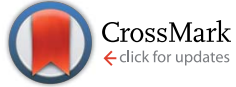

Cite this: J. Anal. At. Spectrom., 2015, 30,828

\title{
An integrated approach based on micro-mapping analytical techniques for the detection of impurities in historical $\mathbf{Z n}$-based white pigments $\uparrow$
}

\author{
V. Capogrosso, ${ }^{\text {a }}$ F. Gabrieli, ${ }^{c}$ S. Bellei, ${ }^{\text {ab }}$ L. Cartechini, ${ }^{c}$ A. Cesaratto, ${ }^{\text {ad }}$ N. Trcera, ${ }^{e}$ \\ F. Rosi, ${ }^{C}$ G. Valentini, ${ }^{a}$ D. Comelli ${ }^{a}$ and A. Nevin ${ }^{b}$
}

In this work we propose an integrated approach, based on synchrotron analysis with micrometric spatial resolution and sub-ppm sensitivity and $\mu$-Raman mapping, for investigating impurities and heterogeneous inclusions in historical samples of $\mathrm{Zn}$-based white pigments. Analysis was performed at the LUCIA beamline at the SOLEIL synchrotron radiation facility for the simultaneous detection of the elemental distribution in suitably prepared pigment samples using micro-X-ray fluorescence $(\mu-X R F)$ mapping and for the investigation of oxidation states and coordination of metals using micro-X-ray near edge absorption ( $\mu$-XANES) spectroscopy. The identification of specific molecular signatures and the detection of their spatial distribution throughout samples by $\mu$-Raman measurements supported and complemented $\mathrm{X}$-ray analysis, allowing the identification of $\mathrm{Cr}$ - and Fe-based inclusions in historical samples. In $\mathrm{ZnO}$ pigments, common impurities are due to the production process and include Fe and, depending on samples, $\mathrm{Cd}, \mathrm{Cl}$ and $\mathrm{Pb}$. In one of the $\mathrm{Zn}$-containing pigments, identified as Lithopone, $\mu$-XRF mapping revealed the presence of $\mathrm{Co}$, both as highly concentrated micrometric inclusions and as impurities throughout the pigment.

Received 3rd November 2014

Accepted 10th February 2015

DOI: $10.1039 / c 4 j a 00385 c$

www.rsc.org/jaas carboxylates in paint. The aim of our work is to apply a method based on complementary analytical spectroscopic mapping techniques on the micrometer scale to study historical samples of Zn-based white pigments from different sources (paint tubes, pastels and from an historic pigment powder). The aim of the approach is to document inclusions and impurities in samples, which can be ascribed to production methods.

There are various reports of the analysis of Zn-based whites using both imaging and spectroscopic techniques. For example, in situ luminescence imaging spectroscopy of a painting by Van Gogh described a peculiar luminescence emission attributed to copper impurities in a $\mathrm{Zn}$-based white pigment. ${ }^{2}$ In another work, spectroscopic investigations on both pure $\mathrm{ZnO}$ and paint models have demonstrated that physical and chemical interactions affect the UV and visible emissions, leading to considerably different optical emissions to those in the pure pigment. ${ }^{3}$ The investigation of both the vibrational and luminescence properties highlights further interactions between semiconductor Zn-based pigments when mixed with the lipidic binder in paint samples and has revealed the formation of chemisorbed carboxylates from the interaction of the lipidic binder and the ZnO surface.

The use of synchrotron-based techniques to investigate original components and degradation products in artist materials has increased significantly during the last ten years. In particular, micro-X-ray fluorescence $(\mu$-XRF) and micro-X-ray near edge absorption ( $\mu$-XANES) techniques are very powerful 
analytical methods in probing the spatial distribution and valence of a given chemical element on the microscopic scale. ${ }^{4-6}$ The chemical characterization of paints on the nanoscale, using high resolution nanoprobe XRF mapping, has shown impurities from $\mathrm{Pb}$ and $\mathrm{Fe}$ ions and has provided insights into both the fabrication and the chemical reactivity of $\mathrm{ZnO}$ pigments. ${ }^{7}$ Photo-luminescent heterogeneities in paint samples containing $\mathrm{ZnO}$ have been also investigated at the DISCO beamline at the SOLEIL synchrotron radiation facility through the use of rasterscanning micro-spectroscopy and full-field micro-imaging. ${ }^{8}$ By combining synchrotron and macroscopic photoluminescence spectroscopy and imaging, it was shown that three historical powder samples of $\mathrm{Zn}$ white pigments were homogeneous on the macroscale yet highly heterogeneous on the micro- and nano-scales. ${ }^{9}$

Our work focuses on the analysis of microscopic heterogeneities in historical samples of $\mathrm{Zn}$-based white pigments from the late $19^{\text {th }}$ and early $20^{\text {th }}$ centuries. After preliminary elemental and molecular characterization of materials using $\mathrm{XRF}$ and micro-Raman spectroscopies, we investigated the presence of trace metal ions in prepared samples by $\mu$-XRF mapping using synchrotron radiation. The high-energy synchrotron X-ray beam provides sensitivities three orders of magnitude higher than that of scanning electron microscopy coupled to energy dispersive X-ray spectroscopy (SEM-EDX), leading to sub-ppm sensitivity. In addition, the oxidation and coordination states of some metallic inclusions (including Fe and $\mathrm{Cr}$ ) have been analysed with $\mu$-XANES spectroscopy. Interpretation of complex data from synchrotron measurements is complemented by the molecular characterization of samples on the $\mu$-scale level with micro-Raman ( $\mu$-Raman) spectroscopy.

\section{Historical background on zinc oxide and lithopone synthesis}

ZnO was formerly used only as a white pigment and was named zinc white. The use of this white pigment spread rapidly and replaced white lead because it had the advantages of being less toxic, ${ }^{\mathbf{1 0}}$ of not darkening in the presence of sulphureous gases and of having better hiding power. Today the term zinc white denotes $\mathrm{ZnO}$ produced by the combustion of metallic $\mathrm{Zn}$ according to the French process (also called indirect process). ${ }^{\mathbf{1 1}, \mathbf{1 2}}$ According to this process, $\mathrm{Zn}$ is used as starting material and volatilized in a special form of retort; the vapour issuing from the retort is oxidized in the presence of air and collected in long settling chambers. The crystallographic and physical properties of the $\mathrm{ZnO}$ can be controlled by adjusting the combustion conditions (flame turbulence and the concentration of oxygen). The typical composition is overall $\mathrm{ZnO}$ (99.69-99.99\%) with small concentrations of $\mathrm{Pb}, \mathrm{Cd}$ and $\mathrm{Fe}^{7,10,12}$ The cost of production of $\mathrm{ZnO}$ from the metal is considerably higher than that of $\mathrm{ZnO}$ produced directly from the ore (American process described below), but it ensures the absence of impurities, such as $\mathrm{Cd}$, which is considerably more volatile than zinc, and produces a brown oxide which leads to the discoloration of the finished product if it is not removed in the process of the manufacture of the metal.

In the United States the largest proportion of $\mathrm{ZnO}$ produced is derived from the ore. In the American process (also called the direct process) the zinc is reduced by the partial combustion of coal and reoxidized at the entrance to the furnace. The direct process is noted for its simplicity, low cost and excellent thermal efficiency, but it produces a less pure form of $\mathrm{ZnO}$ (purity $>98.5 \%$ ) with possible impurities of $\mathrm{Pb}, \mathrm{Cd}, \mathrm{Fe}, \mathrm{S}, \mathrm{Cu}$, and $\mathrm{Mn} .^{7,10,11}$

A third industrial production process is known as the wet process. $\mathrm{ZnO}$ is produced industrially from purified solutions of $\mathrm{ZnSO}_{4}$ or $\mathrm{ZnCl}_{2}$ by precipitating $\mathrm{ZnCO}_{3}$, which is then washed, filtered and finally calcined. This method produces a grade of ZnO with a specific surface area. ${ }^{11}$ The product obtained from this method is known as "precipitated zinc white".

Another white Zn-based pigment is based on $\mathrm{ZnS}$, and was first developed and patented in 1850 in France. ${ }^{13}$ This white pigment, with the largest sales volume, is lithopone, which is a generic name for a white pigment produced through coprecipitation and calcination of $\mathrm{ZnS}$ and $\mathrm{BaSO}_{4}$. Despite the cheapness of its manufacturing processes, and its good properties as a pigment (for example, its hiding power is better than that of zinc oxide, and it shows little interaction with other artistic pigments), it has the objectionable property of degrading upon exposure to UV light, darkening and turning grey after exposure to daylight. For this reason, lithopone never earned a good reputation as an artists' pigment. Nevertheless, as Capua argues, ${ }^{\mathbf{1 4}}$ lithopone was likely used as a cheap extender for other white pigments such as ZnO. Starting from 1928, small quantities of Co (from 0.02 to 0.5 parts per thousand) were added prior to calcination of the crude lithopone, and this was found to prevent the discoloration of the pigment under sunlight. ${ }^{15,16}$

\section{Experimental details}

\section{Material and sample preparation}

The four Zn-based samples presented in this work are a selection from a wider collection of historical white pigment samples

Table 1 Summary of the results obtained from preliminary laboratory measurements

\begin{tabular}{|c|c|c|c|}
\hline Samples & Bulk-XRF & Raman & Grain and agglomerate size \\
\hline WN1 & $\mathrm{Zn}(\mathrm{Fe}, \mathrm{Ba}, \mathrm{Ca}, \mathrm{Cr}, \mathrm{Sn})$ & $\mathrm{ZnO}$ & $100-500 \mathrm{~nm} ; 0.7-1 \mu \mathrm{m}$ \\
\hline LF1 & $\mathrm{Zn}(\mathrm{Fe}, \mathrm{Pb}, \mathrm{Ba}, \mathrm{Ca})$ & $\mathrm{ZnO}+$ lipidic component & $200-500 \mathrm{~nm} ; 0.5-1 \mu \mathrm{m}$ \\
\hline LF2 & $\mathrm{Zn}(\mathrm{Fe}, \mathrm{Pb}, \mathrm{S}, \mathrm{Cr})$ & $\mathrm{ZnO}, \mathrm{ZnCO}_{3}$ & $0.2-1 \mu \mathrm{m}$ \\
\hline LF3 & $\mathrm{Zn}, \mathrm{Ba}(\mathrm{Sr}, \mathrm{S}, \mathrm{Pb}, \mathrm{Fe}, \mathrm{Ca})$ & Lithopone + lipidic component & $0.5-1 \mu \mathrm{m}$ \\
\hline
\end{tabular}


and are representative of the different artist materials available at the turn of the $20^{\text {th }}$ C.: pastels, paints and the pigments from which they were made.

One sample is from a paint tube of Chinese white Winsor \& Newton from the Courtauld Institute of Art in London (WN1). In 1834 Winsor \& Newton developed a new method of heating the $\mathrm{ZnO}$ to increase its opacity. ${ }^{17}$ This new type of $\mathrm{Zn}$ white was called Chinese white.

The remaining three samples have been drawn from the well known French manufacturer, Lefranc-Bourgeois, which, at the beginning of the $20^{\text {th }}$ century, was the largest producer of $\mathrm{ZnO}$ in Europe.

The first Lefranc sample is a small white pastel fragment from a collection of Lefranc-Bourgeois Raffaelli pastels (LF1). The second sample (LF2) was supplied as a powder by the Colart Lefranc factory, and is a Lefranc watercolour pigment, from a bottle labelled Blanc de Neige, Vieille-Montagne Mars, Le Mans (1893). The last sample comes from a Lefranc paint tube belonging to the collection of Grubicy-Benvenuti of the Livorno Foundation (Livorno, Italy) (LF3).

Preliminary XRF and $\mu$-Raman analysis were performed on samples without any specific preparation in order to identify the pigment and binder composition.

Scanning electron microscopy was carried out on samples coated with a thin layer of gold to gain information on grain morphology. For $\mu$-X-ray synchrotron and $\mu$-Raman mapping measurements, small quantities of samples (10 $\mathrm{mg}$ on average) were pressed as pellets of $0.5 \mathrm{~cm}$ diameter on a boron nitride substrate. During sample preparation we aimed to avoid contamination through proper handling, but we cannot exclude previous contamination prior to sampling of the four pigments.

\section{Experimental apparatus}

X-ray fluorescence spectroscopy. Laboratory measurements on samples were carried out using a portable XRF spectrometer (Elio, XGLab srl). The instrument is a fast system with a large area silicon drift detector (SDD) $\left(25 \mathrm{~mm}^{2}\right)$, and it is particularly efficient due to the X-ray transmission generator that can reach up to $50 \mathrm{kV}$ and to a high solid detection angle geometry. The excitation source works with a Rh anode and the beam is collimated to a spot diameter on the sample surface of about 1.3 mm. XRF measurements have been carried out by fixing the tube voltage at $40 \mathrm{kV}$ and $15 \mathrm{kV}$ exploring a field of analysis from 1 to $30 \mathrm{keV}$.

Scanning electron microscopy. The scanning electron microscope (SEM) employed was a Philips XL30 instrument equipped with a $\mathrm{LaB}_{6}$ source and an EDAX/DX4 detector. The acceleration potential voltage was maintained at $15 \mathrm{kV}$, the magnification was held at $20000 \times$. For the sake of concision, secondary electron (SE) images of samples are reported in the ESI. $\dagger$

Raman spectroscopy. $\mu$-Raman measurements were performed using a laboratory JASCO NRS-3100 spectrophotometer equipped with a diode laser at $785 \mathrm{~nm}$, a grating of 800 lines per $\mathrm{mm}$, an optical microscope and a charge coupled device (CCD) cooled to $-50{ }^{\circ} \mathrm{C}$ with a Peltier cooling system. The areas of (a) $\mathrm{WN} 1$

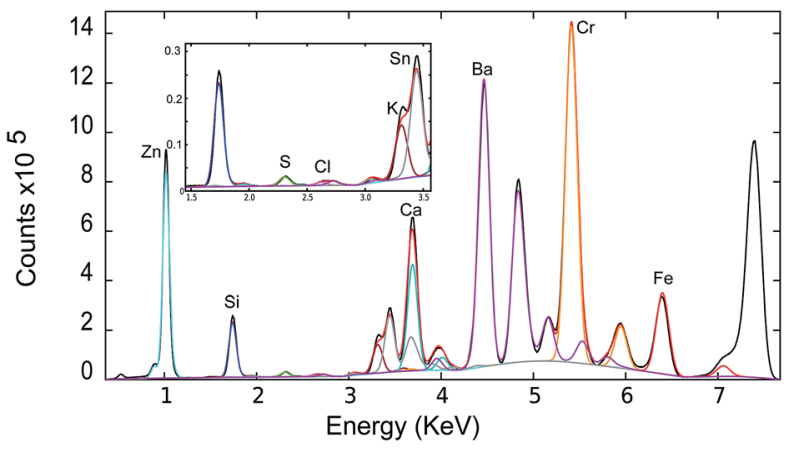

(b) LF1

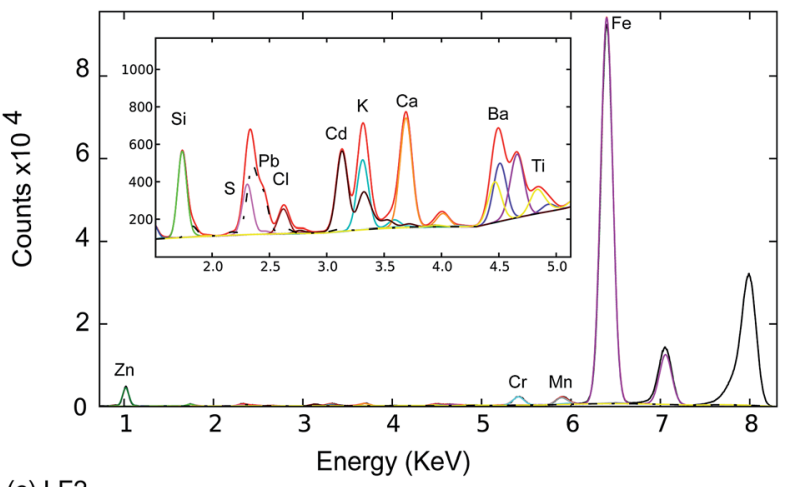

(c) LF2

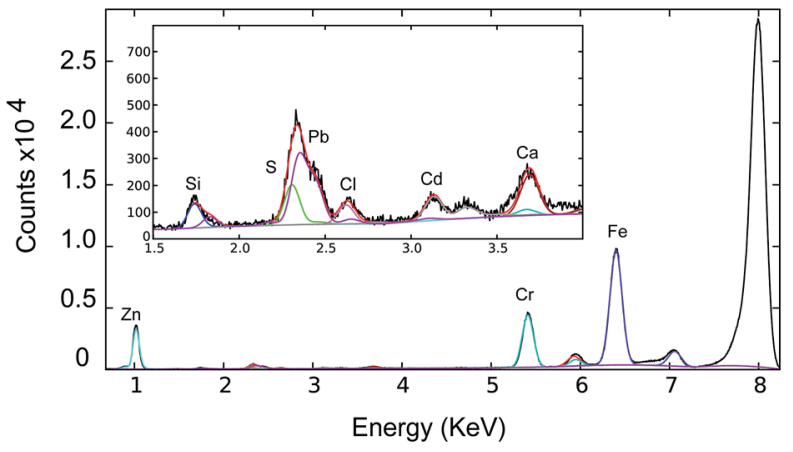

(d) LF3

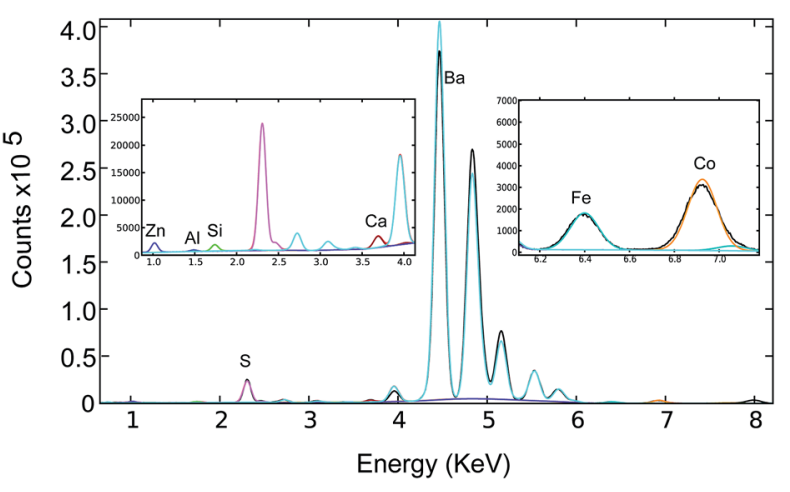

Fig. $1 \mu$-XRF cumulated spectra, obtained as the sum in all points of a mapped area $100 \times 100 \mu \mathrm{m}^{2}$ in size (black line), for the four samples. The result of the fitting of the cumulative spectrum with PyMca software is shown by the red line. In the insets, magnifications of energy regions are shown in order to highlight trace elements. 
Table 2 Summary of the results obtained from $\mu$-XRF and $\mu$-Raman techniques

\begin{tabular}{llll}
\hline Samples & $\mu$-XRF (impurities) & $\mu$-XRF (inclusions) & $\mu$-Raman \\
\hline WN1 & K & Ba, Cr, Ca, Si, Cl, Fe & BaCrO $_{4}$ \\
LF1 & Pb, Cd, Fe, Al, K, S, & Fe, Cr, Mn, Ti, Ba & FeO $(\mathrm{OH})$ \\
& Cl, Ca, Si & & \\
LF2 & Cl, Fe, Pb, S, Cd, Ca, Si & Cr, Fe & \\
LF3 & $\mathrm{Ba}, \mathrm{S}, \mathrm{Al}, \mathrm{Si}, \mathrm{Co}$ & $\mathrm{Co}, \mathrm{Ca}, \mathrm{Fe}$ &
\end{tabular}
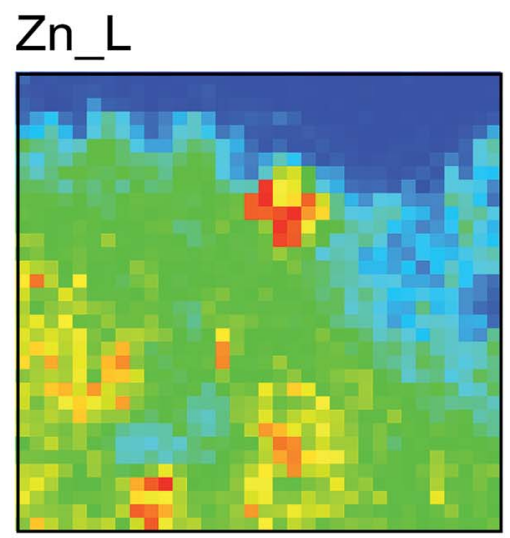

Si_K
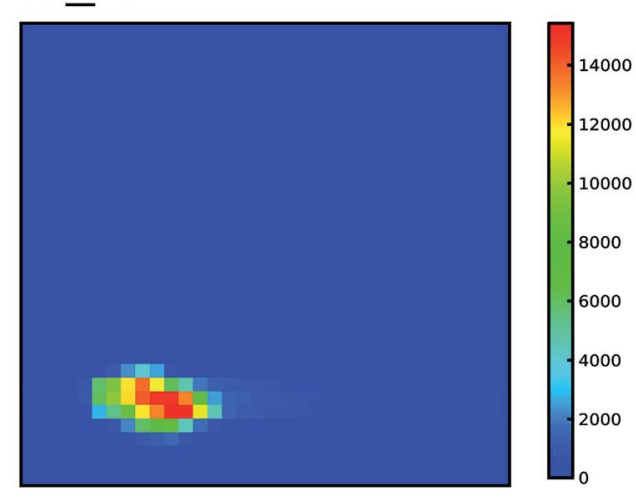

\section{$\mathrm{Ca} \mathrm{K}$}
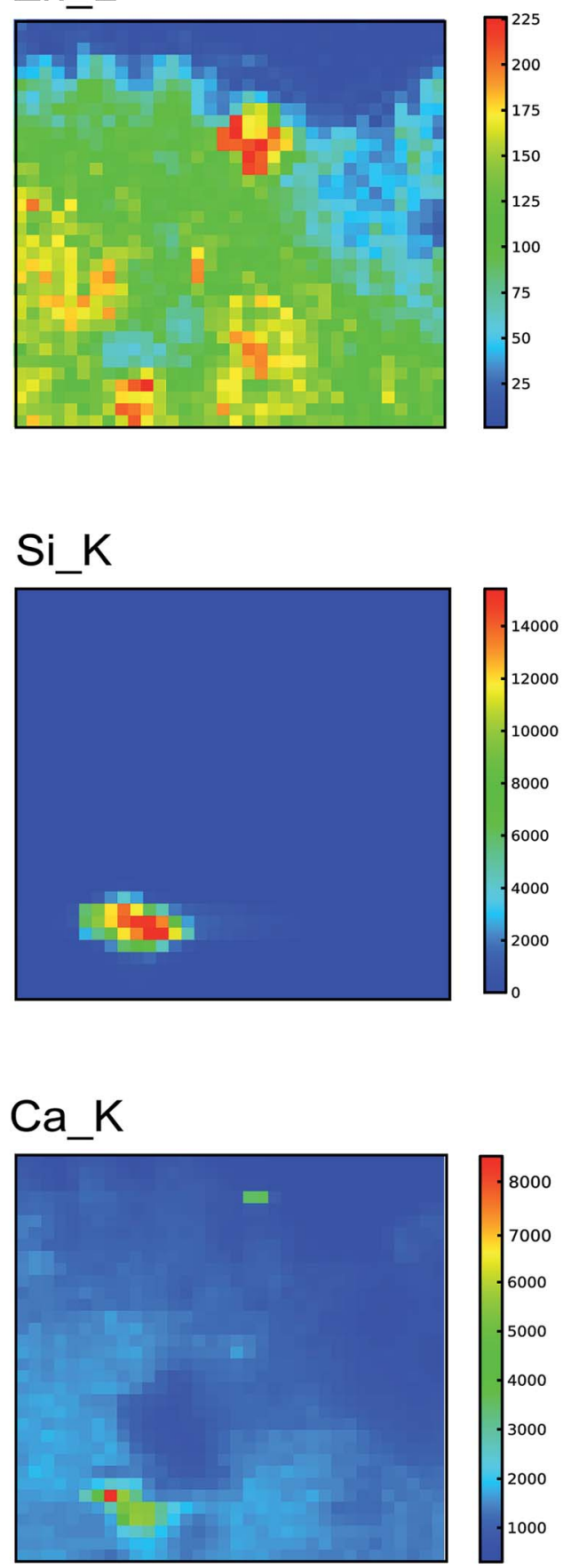

interest were focused with the objective $100 \times$ and the spectra were recorded in the range $180-1800 \mathrm{~cm}^{-1}$ with a spectral

For preliminary measurements on samples the exposure time varied from 10 to $20 \mathrm{~s}$ and the number of accumulations from 10 to 15; for mapping of pellets exposure times varied from 5-10 s with 10 accumulations. The laser power was changed from $1 \mathrm{~mW}$ to a maximum of $18 \mathrm{~mW}$ on the sample.

Synchrotron-based X-ray spectroscopy. Simultaneous detection of the atomic distribution and the oxidation state of some resolution of about $2 \mathrm{~cm}^{-1}$ and a beam spot size of $3 \mu \mathrm{m}$.
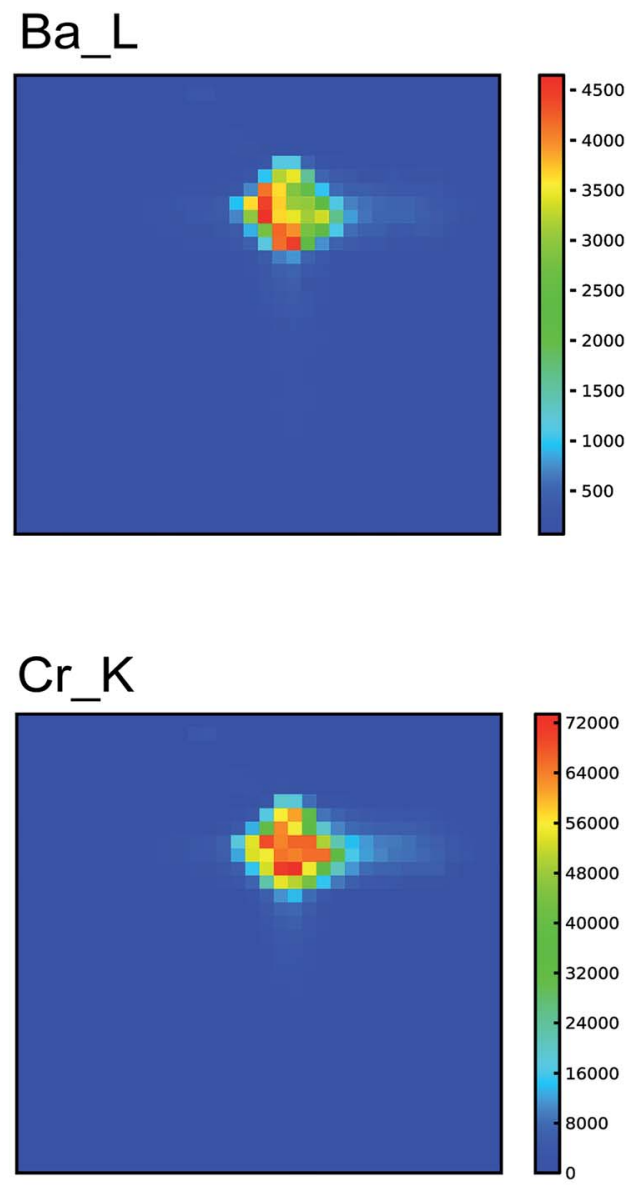

\section{Cl_K}

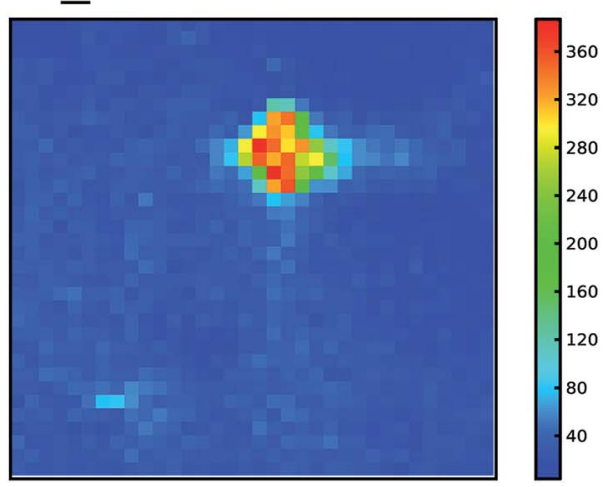

Fig. 2 WN1 sample: $\mu$-XRF maps showing the spatial distribution of Zn, Ba, Si, Cr, Ca and Cl over an area of $100 \times 100 \mu m^{2}$ with a step of $3 \mu \mathrm{m}$. 
specific heterogeneities was carried out using the set-up at the LUCIA beamline at SOLEIL. ${ }^{18}$ The LUCIA beamline is a tender (0.8-8 keV) X-ray microprobe able to detect chemical speciation and to carry out elemental mapping by $\mu$-XANES and $\mu$-XRF spectroscopy respectively. The instrument uses a photon source based on an undulator of the APPLE-II type and a double crystal monochromator for selecting X-ray energy. Analysis was performed in a low vacuum chamber in which samples were placed on a micro-positioning $(x, y, z)$ sample stage. Fluorescence spectra were collected using a 4-element SDD detector. Representative $\mu$-XRF maps of $1000 \times 1000 \mu \mathrm{m}^{2}$ with steps of $50 \mu \mathrm{m}$ were collected in order to identify potential heterogeneities. Afterwards, focused areas containing heterogeneities were chosen and $\mu$-XRF maps of $100 \times 100 \mu \mathrm{m}^{2}$ with a step size of 3 $\mu \mathrm{m}$ were collected using a beam spot of $3 \times 3 \mu \mathrm{m}^{2}$, and with an excitation energy between 7.4 and $8 \mathrm{keV}$ for the analysis of different elements. At this energy only X-ray fluorescence L-lines of $\mathrm{Zn}$ at $1.0 \mathrm{keV}$ are excited and used for detection. $\mu$-XANES spectra were acquired by simultaneously measuring the fluorescence yield (FY) and the total electron yield (TEY), but results are reported only for FY. XANES spectra were collected from inclusions for the Fe K-edge and Cr K-edge.

XRF data were processed using the PyMca software, which has also been used to obtain fluorescence maps. ${ }^{19}$ Single element imaging was performed setting Regions Of Interest (ROI) around the characteristic element peaks. In case of overlapping peaks due to the superposition of different lines, it was necessary to fit all spectra separately in order to differentiate contributions and for the reconstruction of an image from the fitted areas using a batch fitting tool. False colour images were generated to represent the presence of different elements in samples, and the scale of each image was chosen to best highlight the heterogeneities detected. Absorption data, after background subtraction, were normalized using Athena. ${ }^{20}$

For the estimation of the size of localized inclusions, the full width at half maximum of a line scan in the $\mu$-XRF or $\mu$-Raman maps was used.

\section{Results}

\section{Preliminary laboratory measurements}

Results of the preliminary laboratory analysis of samples are summarised in Table 1 . All samples contain $\mathrm{Zn}$, as detected with XRF.

In preliminary XRF spectra from WN1, traces of $\mathrm{Fe}, \mathrm{Ba}, \mathrm{Ca}$, $\mathrm{Cr}$ and $\mathrm{Sn}$ were detected. Raman spectra identified the pigment as $\mathrm{ZnO}$ due to bands at 438 (vs), 383 (w) and $331(\mathrm{w}) \mathrm{cm}^{-1} \cdot{ }^{16}$ The sample is characterized by submicron grains in the range of 100-500 nm with a nodular shape typical of fast burning synthesis, which suggests a thermal production process. ${ }^{13}$ Few larger conglomerates of about $0.7-1 \mu \mathrm{m}$ with subhedral hexagonal shape are also visible in SEM images.

XRF measurements on LF1 highlight minor quantities of Fe and traces of $\mathrm{Pb}, \mathrm{Ba}$ and $\mathrm{Ca}$. Raman spectra confirmed the presence of $\mathrm{ZnO},{ }^{21}$ and bands between 1440 and $1060 \mathrm{~cm}^{-1}$ indicate the presence of a lipidic component, ascribed to the binder. ${ }^{22}$ Indeed, the presence of a lipid binder hinders the observation of the grain morphology in SEM due to the incorporation of the pigments in an amorphous matrix. However, few polyhedral shaped conglomerates with dimensions of 0.5-1 $\mu \mathrm{m}$ are visible. Furthermore, needle-like shapes of about 200-500 $\mathrm{nm}$ are present around the large conglomerates.

LF2 has traces of $\mathrm{Fe}, \mathrm{Pb}, \mathrm{S}$ and $\mathrm{Cr}$. From $\mu$-Raman analysis the sample has been identified as a mixture of $\mathrm{ZnO}$ and $\mathrm{ZnCO}_{3}$. The pigment grains have variable dimensions, in the range of $0.2-1 \mu \mathrm{m}$, and morphology varying from large polycrystalline angular conglomerates to smaller prismatic or acicular crystallites. This morphology can be explained by a non-controlled slow crystallization process, such as the wet process. This production method is in agreement with the presence of carbonates, as described in the historical background section. ${ }^{\mathbf{1 1}}$

In contrast to the three $\mathrm{ZnO}$ samples (WN1, LF1 and LF2), XRF on LF3 highlighted the presence of $\mathrm{Ba}$ in addition to $\mathrm{Zn}$, with weaker signals of $\mathrm{Sr}$ and $\mathrm{S}$ along with traces of $\mathrm{Pb}, \mathrm{Fe}$ and $\mathrm{Ca} . \mu-$
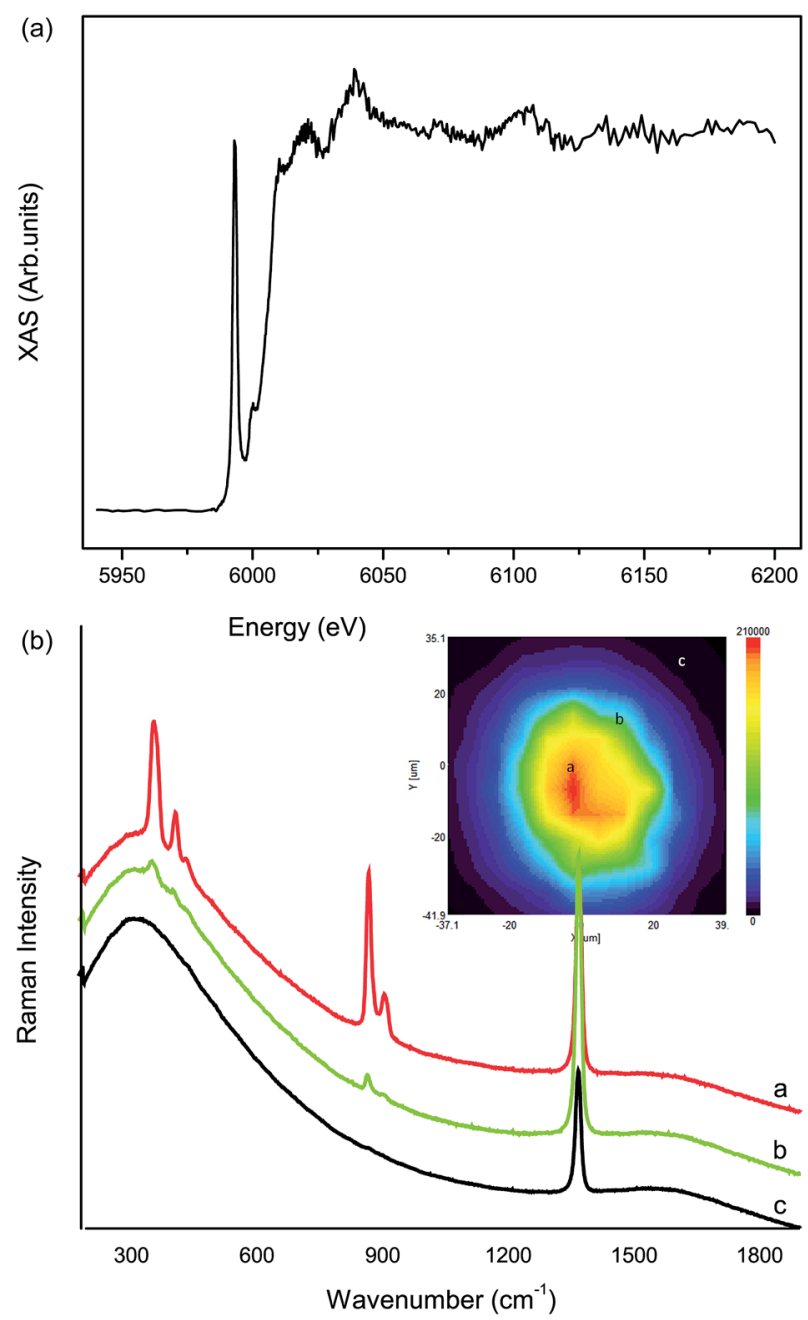

Fig. 3 WN1 sample: (a) $\mu$-XANES spectrum at $\mathrm{Cr} \mathrm{K}$-edge recorded from the inclusion highlighted in the $\mathrm{Cr}$ map shown in Fig. 2; (b) $\mu$ Raman map, resulting from the integration of the chromate band at $862 \mathrm{~cm}^{-1}$ over an area of $77 \times 77 \mu \mathrm{m}^{2}$, and $\mu$-Raman spectrum registered at the core of the inclusion (a), at the border (b) and outside the inclusion (c). 
Raman spectra of the powdered sample showed peaks of $\mathrm{BaSO}_{4}$ and a weak band at $348 \mathrm{~cm}^{-1}$, assigned to $\mathrm{ZnS} .{ }^{21}$ The spectrum is consistent with the reference spectra of lithopone. The observation of additional bands at $1440 \mathrm{~cm}^{-1}$ and $1297 \mathrm{~cm}^{-1}$ suggests the presence of a lipidic component. ${ }^{22}$ Few polyhedral shaped conglomerates with dimensions of $0.5-1 \mu \mathrm{m}$ are visible in SEM images due to the presence of the lipidic binder.

From bulk XRF measurements, a common trace element present in all four samples is Fe, which could be ascribed to the use of the raw material sphalerite ( $\mathrm{Zn}, \mathrm{Fe}) \mathrm{S}$ in synthesis. This cubic resinous sulphide mineral is the most common of the zinc minerals, and it is nearly always associated with $\mathrm{Al}$, resulting from acidic weathering of rock and sulphide minerals, and may also include $\mathrm{Pb}$, resulting from mine drainage activity. ${ }^{23}$

\section{$\mu$-XRF and $\mu$-Raman mapping}

As it will be shown hereafter for the considered samples, the $\mu$-XRF and $\mu$-Raman combined approach allows us to distinguish amongst elements homogeneously distributed throughout the pigment matrix and elements spatially localized in a confined area. In the text, the former and the latter will be referred to with the notation of impurities and inclusions, respectively. Moreover, with the combination of the two $\mu$-mapping techniques we can further obtain insights into the size of localized inclusions. This information can provide understanding of the origin of trace metals in samples.

\section{WN1}

In Fig. 1a the cumulative spectrum from $\mu$-XRF mapping using synchrotron radiation, fitted using PyMca, is reported. $\mu-\mathrm{XRF}$ measurements highlight the presence of $\mathrm{Ba}$ and $\mathrm{Cr}$, combined with traces of $\mathrm{Ca}$, Fe and Si. Weak emissions are assigned to K, $\mathrm{S}$, and $\mathrm{Cl}$. $\mu$-XRF maps of compositional distribution provided evidence of the spatial correlation of different elements, allowing the differentiation between homogeneously distributed impurities and localized inclusions, as summarized in Table 2 for all samples.

$\mu$-XRF maps (Fig. 2) revealed the spatial correlation between $\mathrm{Si}$ and $\mathrm{Ca}$ in an asymmetric agglomerate inclusion estimated as $21 \times 12 \mu^{2}$ in size. No molecular identification has been achieved for these inclusions; the spatial correlation may be an indication of possible calcium containing silicates, but other compounds cannot be excluded.

An excellent spatial correlation between $\mathrm{Ba}, \mathrm{Cr}$ and $\mathrm{Cl}$ distributions is seen in an agglomerate $20 \mu \mathrm{m}$ in diameter. In order to clarify the oxidation state of the $\mathrm{Cr}$ in the inclusion,
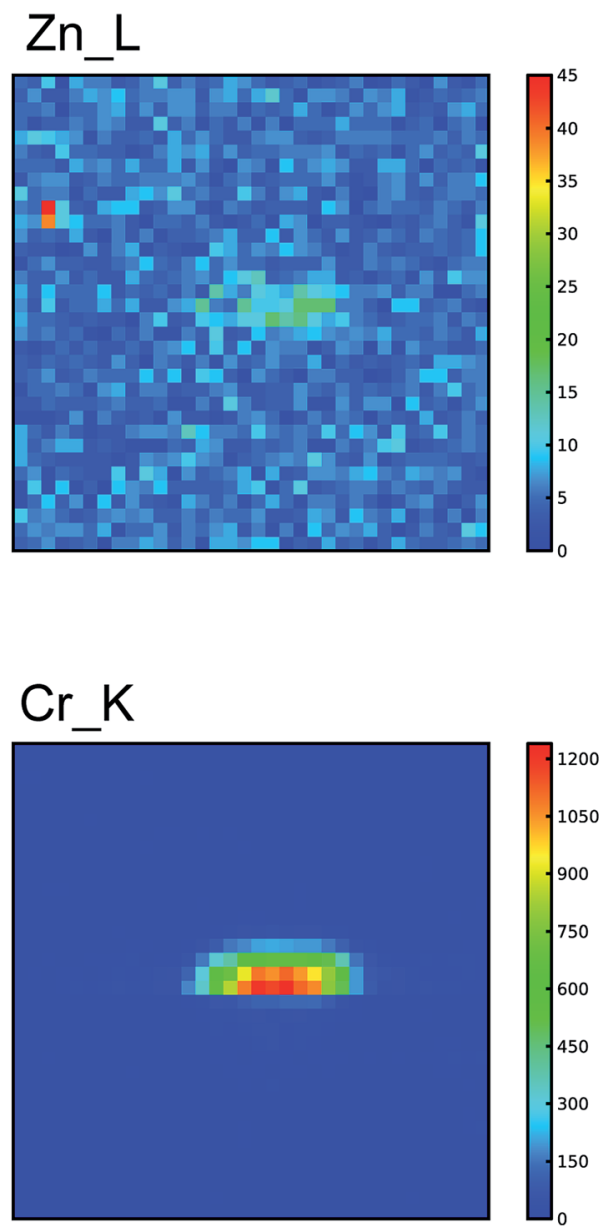
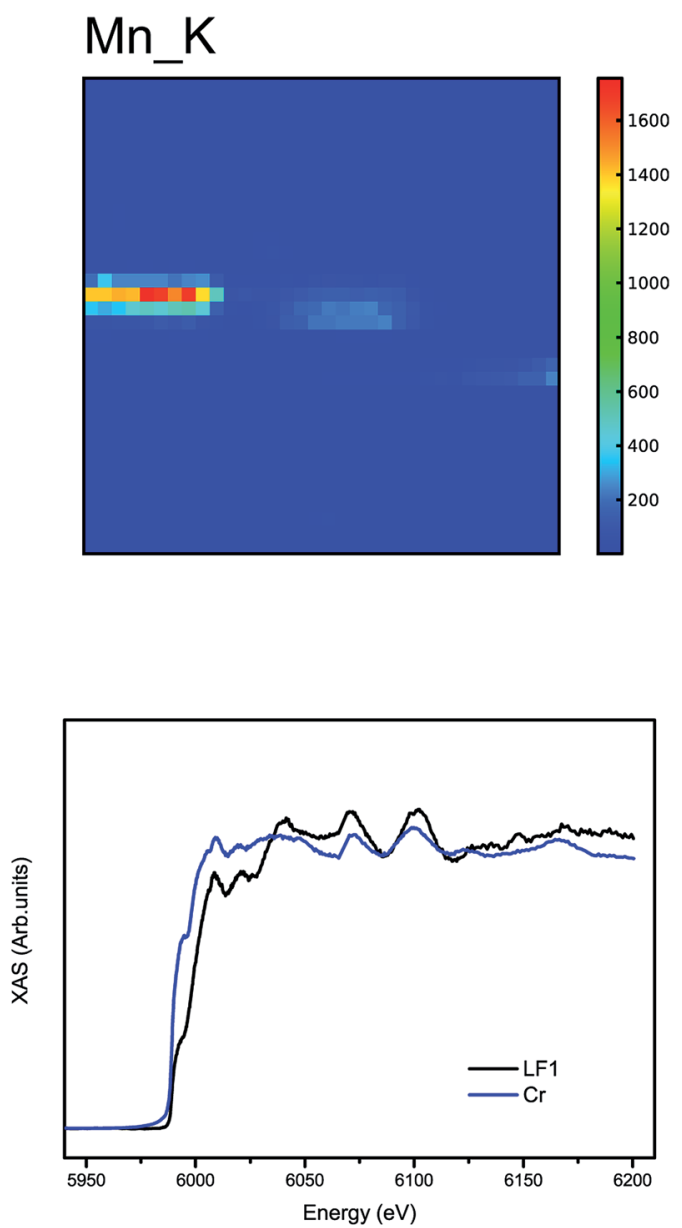

Fig. 4 LF1 sample: (a) $\mu$-XRF maps showing the spatial distribution of $\mathrm{Zn}, \mathrm{Mn}$ and $\mathrm{Cr}$ over an area of $100 \times 100 \mu \mathrm{m}^{2}$ with a step of $3 \mu \mathrm{m}$. On the bottom right, $\mu$-XANES spectra recorded from the $\mathrm{Cr}$ inclusion (black) and a reference sample of metallic $\mathrm{Cr}$ (blue). 
$\mu$-XANES measurements have been performed on the same area. The measured Cr K-edge $\mu$-XANES spectrum (Fig. 3a) shows a well-defined pre-edge peak at $5.993 \mathrm{keV}$, typical of $\mathrm{Cr}(\mathrm{vI})$ compounds with a tetrahedral coordination geometry. This peak corresponds to a bound state $1 \mathrm{~s}$ to $3 \mathrm{~d}$ dipole-forbidden transition, and it is related to the non-centrosymmetric nature of the tetrahedral structure that favours a strong hybridization of the $\mathrm{Cr} 3 \mathrm{~d}$ and $\mathrm{O} 2 \mathrm{p}$ orbitals. ${ }^{24}$ The spectrum is comparable with that of $\mathrm{BaCrO}_{4}$ reported by Monico. ${ }^{6}$

The presence of $\mathrm{BaCrO}_{4}$ in sample WN1 is confirmed by $\mu$ Raman spectra acquired from yellow crystals (of about $45 \times 45$ $\mu \mathrm{m}^{2}$ ) visible on the pellet investigated at the synchrotron. An example image of a $\mathrm{BaCrO}_{4}$ crystal from $\mu$-Raman mapping is reported in Fig. $3 \mathrm{~b}$. The map is $77 \times 77 \mu \mathrm{m}^{2}$ and was obtained by acquiring each spectrum with a step of $7 \mu \mathrm{m}$. The false-colour image was obtained by integration of the band relative to the symmetric stretching mode of the $\mathrm{CrO}_{4}{ }^{2-}$ group at $862 \mathrm{~cm}^{-1} .^{21}$

In Fig. 3b, the Raman spectrum recorded at the center of the inclusion (a) clearly shows bands distinctive to $\mathrm{BaCrO}_{4}$, including the symmetric stretching modes at 862 and $900 \mathrm{~cm}^{-1}$ as well as the bending at $403 \mathrm{~cm}^{-1}$. As expected these diagnostic bands diminish in intensity when moving towards the boundaries of the inclusion (spectra $b$ and $c$ in Fig. 3b) where the boron nitride signal from the substrate is mainly present at $1368 \mathrm{~cm}^{-1}$.

$\mathrm{BaCrO}_{4}$ can be synthesized by co-precipitating barium hydroxide with potassium chromate $\left(\mathrm{Ba}(\mathrm{OH})_{2}+\mathrm{K}_{2} \mathrm{CrO}_{4} \rightarrow\right.$ $\left.\mathrm{BaCrO}_{4}+2 \mathrm{KOH}\right)$ or, alternatively, by the interaction of barium chloride $\left(\mathrm{BaCl}_{2}\right)$ with sodium chromate $\left(\mathrm{Na}_{2} \mathrm{CrO}_{4}\right){ }^{25,26}$ This synthesis method would explain the presence of $\mathrm{Cl}$ in the same inclusion. $\mathrm{BaCrO}_{4}$ is usually associated with lemon yellow, and in WN1 this is possibly an unintentional contamination introduced during the mixing of the pigments in the paint.

\section{LF1}

$\mu$-XRF measurements on LF1 are shown in Fig. 1b. LF1 is characterised by the presence of $\mathrm{Fe}$, and to a lesser extent $\mathrm{Cr}$ and $\mathrm{Mn}$, with traces of $\mathrm{Al}, \mathrm{Si}, \mathrm{S}, \mathrm{Pb}, \mathrm{Ba}, \mathrm{Cl}, \mathrm{Cd}, \mathrm{K}$ and Ti.

$\mathrm{Pb}$ and $\mathrm{Cd}$, distributed homogeneously throughout the $\mathrm{ZnO}$ matrix, are ascribed to residues of the production process. The presence of Cd may exclude the French process in the synthesis of the sample. Ti, which appears as localized inclusions, was probably due to $\mathrm{TiO}_{2}$ added to the $\mathrm{ZnO}$ matrix as a whitening agent.

$\mathrm{Zn}, \mathrm{Mn}$ and $\mathrm{Cr} \mu$-XRF maps obtained on an area of $100 \times 100$ $\mu \mathrm{m}^{2}$ are reported in Fig. 4, with $\mathrm{Mn}$ and $\mathrm{Cr}$ appearing as inclusions which are not spatially correlated with one another.

Regarding the presence of $\mathrm{Mn}$ inclusions $\left(30 \times 3 \mu^{2}\right.$ in size), it has been reported that Mn oxides (in particular pyrolusite) and dehydrated Mn salts were added in small proportions to paint tube formulations in order to improve the siccative properties of lipid binders. ${ }^{27} \mathrm{~A}$ similar explanation may account for the presence of $\mathrm{Al}$; in fact aluminum stearate was a common additive to paints as an emulsifier. ${ }^{1}$

To better understand the presence of the Cr inclusion $(30 \times$ $10 \mu \mathrm{m}^{2}$ in size), $\mu$-XANES measurements at the Cr K-edge were collected. The spectrum reported in Fig. $4 \mathrm{~d}$ is comparable with that of a metallic $\mathrm{Cr}$ reference. The presence of metallic Cr could be explained by fly ash (mineral residue resulting from the combustion of coal in electric generating plants and in reducing environments) during the production process, so, in this sample the wet process is excluded and we hypothesise that this sample was produced by the American process.

Significant concentrations of Fe were mapped on different areas of the sample, with the element found both as a localized heterogeneity and homogeneously distributed throughout the sample matrix. In the latter case, its presence can be explained as a residue of the production process, whereas in the former it is likely due to sample contamination. As an example, in an area with a concentrated $\mathrm{Fe}$ inclusion $\left(9 \times 12 \mu \mathrm{m}^{2}\right.$ in size $)$, the $\mu$-XANES spectrum at the Fe K-edge has been measured (Fig. 5a). The spectrum is comparable with an iron oxide reference. $^{28} \mu$-Raman measurements identified this Fe-based
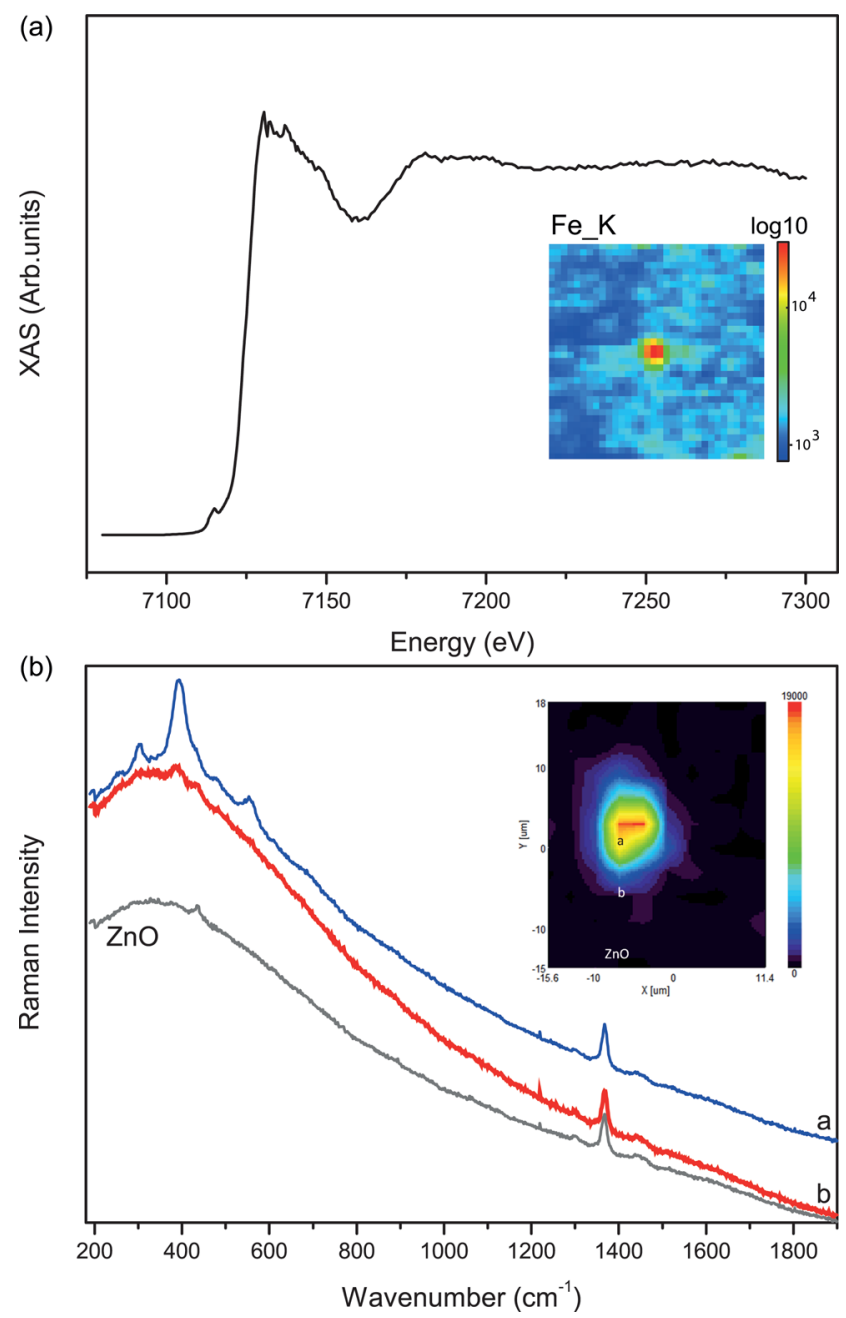

Fig. 5 LF1 sample: (a) $\mu$-XANES spectrum at the Fe K-edge acquired from an area with a concentrated Fe inclusion seen in the $\mu$-XRF map shown in the inset on a logarithmic scale; (b) $\mu$-Raman map acquired on the same Fe inclusion, resulting from the integration of the band at $389 \mathrm{~cm}^{-1}$ over an area of $36 \times 30 \mu \mathrm{m}^{2}$, and micro-Raman spectrum registered at the core (a) and at the border of the inclusion (b) and on a $\mathrm{ZnO}$ reference sample. 
compound as goethite $(\alpha-\mathrm{FeO}(\mathrm{OH}))$ by the presence of three bands at $300(\mathrm{~m}), 389(\mathrm{~s})$ and $550(\mathrm{~m}) \mathrm{cm}^{-1}$, related to the vibrational modes of the $\mathrm{Fe}-\mathrm{O}$ and $\mathrm{Fe}-\mathrm{OH}$ bonds. ${ }^{29}$

$\mu$-Raman mapping has been carried out on the same area analysed by $\mu$-XANES, scanning a surface of $36 \times 30 \mu \mathrm{m}^{2}$ with a 3 $\mu \mathrm{m}$ step size. The chemical image shown in Fig. $5 \mathrm{~b}$ has been obtained by integration of the most intense band at $389 \mathrm{~cm}^{-1}$, and suggests the dimensions of the $\alpha-\mathrm{FeO}(\mathrm{OH})$ inclusion to be approximately $10 \times 10 \mu \mathrm{m}^{2}$, in good agreement with $\mu$-XRF data.

\section{LF2}

In Fig. 1c the $\mu$-XRF spectrum of LF2 is dominated by the presence of $\mathrm{Fe}$ and $\mathrm{Cr}$ with traces of $\mathrm{S}$ and $\mathrm{Pb}$. The micrometric maps also highlight the presence of $\mathrm{Si}, \mathrm{Ca}, \mathrm{Cd}$ and $\mathrm{Cl}$.

This sample is primarily of interest because it is representative of the wet process, as reported above. The identification of traces of $\mathrm{Cl}$ and its homogeneous distribution throughout the sample matrix, as shown in the $\mu$-XRF Cl map (Fig. 6), suggest the use of a $\mathrm{ZnCl}_{2}$ solution as a precursor in the wet process.

Homogeneously distributed impurities of $\mathrm{Fe}, \mathrm{Pb}$, and $\mathrm{Cd}$ are typical residues of this production process. ${ }^{10} \mathrm{Fe}$ was detected both homogeneously distributed throughout the sample matrix and as localized heterogeneities. $\mathrm{Cr}$ is found as an inclusion (20 $\mu \mathrm{m}$ in diameter), and it is reasonable that it has been unintentionally added to the pigment. In contrast to $\mathrm{WN} 1$, no Ba was detected in LF2, and hence the metal could be associated with a different Cr-based pigment.

$\mathrm{Ca}$ and $\mathrm{Si}$ are homogeneously distributed in the sample and were probably added as extenders during the production process.

\section{LF3}

The cumulative $\mu$-XRF spectrum is reported in Fig. $1 d$, and it is dominated by the presence of $\mathrm{Ba}$ and $\mathrm{S}$.

The investigation of the sample with a micrometric resolution allowed the mapping of the presence of particles containing $\mathrm{Al}, \mathrm{Si}, \mathrm{Ca}, \mathrm{Fe}$ and traces of Co. The small amount of $\mathrm{Si}$ homogeneously distributed in the matrix is likely due to the presence of an extender. Similarly to the other lipid-based sample (LF1), the presence of Al in LF3 is likely due to the presence of an aluminum stearate in the paint tube formulation.

$\mu$-XRF maps reported in Fig. 7 show a strong correlation between the distributions of $\mathrm{Ba}, \mathrm{S}$ and $\mathrm{Zn}$. This confirms the results from $\mu$-Raman measurements that identified the pigment as lithopone.

Finally, the Co map (shown in Fig. 7 with a logarithmic scale) highlights the presence of both highly concentrated cobalt

\section{$\mathrm{Zn} L$}

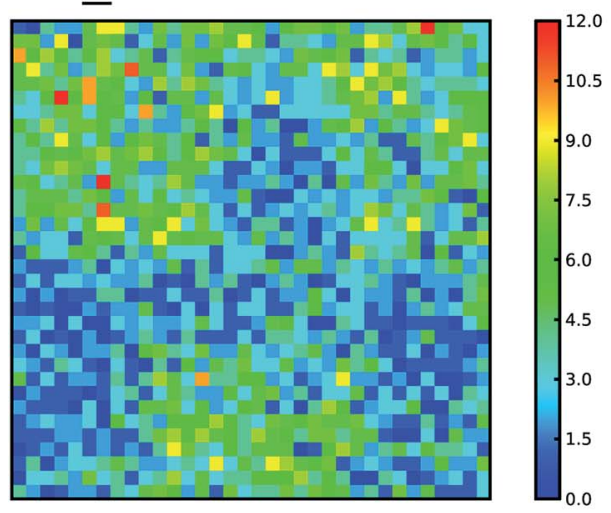

\section{Fe_K}
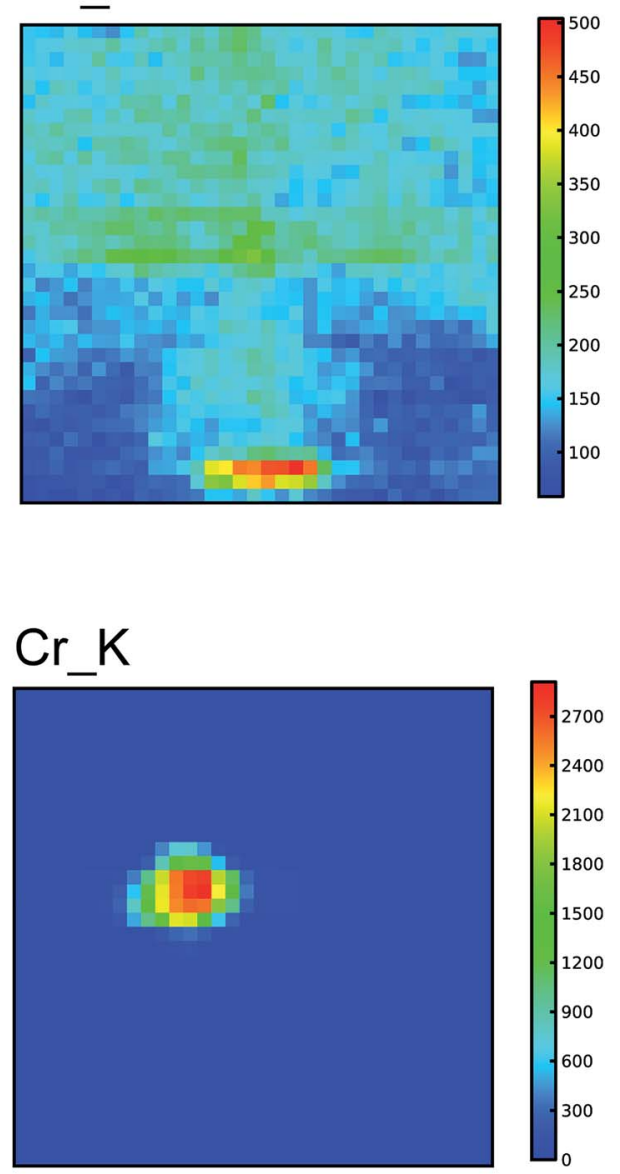

Fig. 6 LF2 sample: $\mu$-XRF maps showing the spatial distribution of $\mathrm{Zn}, \mathrm{Fe}, \mathrm{Cl}$ and $\mathrm{Cr}$ over an area of $100 \times 100 \mu \mathrm{m}^{2}$ with a step of $3 \mu \mathrm{m}$. 

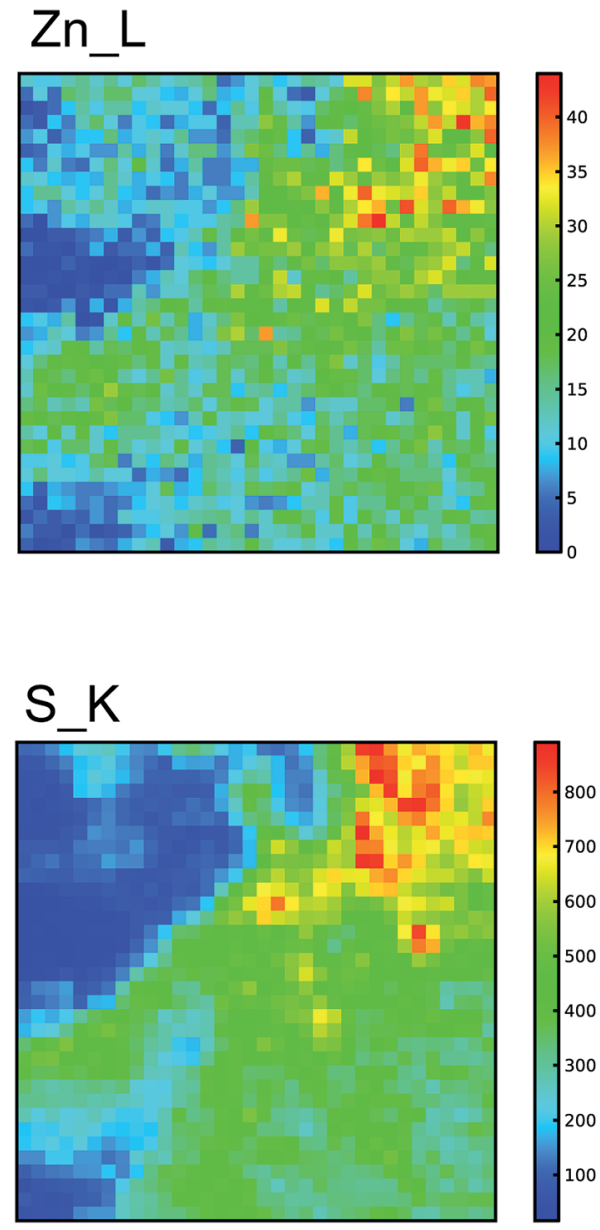

Fig. 7 LF3 sample: $\mu$-XRF maps showing the spatial distribution of Zn, Ba, S and Co (in logarithmic scale only for Co) over an area of $100 \times 100$ $\mu \mathrm{m}^{2}$ with a step of $3 \mu \mathrm{m}$.

micrometric inclusions together with the homogeneous distribution of trace Co correlated with $\mathrm{Zn}$ and $\mathrm{Ba}$, suggesting that the paint tube was produced after 1928, as explained in the introduction. ${ }^{15,16}$ This information suggests that the paint tube was not part of the collection of Vittore Grubicy de Dragon (1851-1920) but rather of Benvenuto Benvenuti (1881-1959).

\section{Discussion}

In the previous section the results obtained from the analysis of historical Zn-based white samples of different origin were reported.

The results demonstrate how bulk analysis with limited sensitivity can yield diagnostic information for the identification of $\mathrm{ZnS}$ or $\mathrm{ZnO}$, as well as data regarding the presence of trace elements, but careful sample preparation and study using micro-analytical techniques is necessary for a better understanding of inclusions and impurities. While morphological information obtained by SEM is useful for the assessment of pigments in powder, the presence of binder (as in the case of paint tube) hinders the observation of grain dimensions or
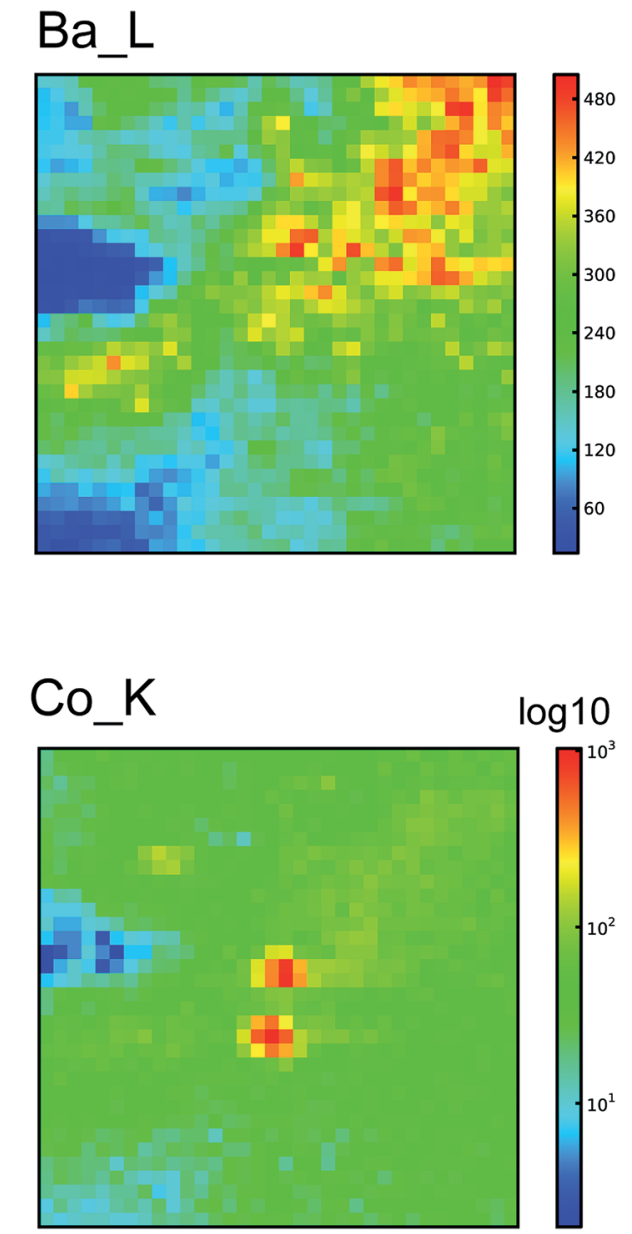
the production of $\mathrm{ZnO}$. On the other hand, the identification of traces of Co in LF3 as an intentional impurity rather than isolated inclusions allows the dating of the lithopone sample to a specific historical period. Establishing production processes is complex, and only in some cases can morphology (as evidenced by SEM) and impurities give convincing proof of a particular production process. Indeed in the four samples studied, while the wet process is clear for LF2 from the particle size, the presence of carbonates and $\mathrm{Cl}$, other production processes are more elusive. In LF1, traces of Cd and metallic Cr suggest the American process, while in the case of WN1 it is only possible from elemental data to exclude the wet process. More in depth investigations of a statistical number of samples of known production type would be required to correlate impurities with a specific production method or geographical origin.

\section{Conclusions}

Our work proposes a multi-analytical methodological approach for the chemical characterization of modern Zn-based pigments with a micrometric resolution. This is achieved by combining $\mu$-XRF, $\mu$-XANES and $\mu$-Raman techniques, and could become a tool for investigating not only a particular class of semiconductor pigments, as in this work, but different modern pigments.

The identification and distinction between homogeneously distributed impurities and localized inclusions in samples allowed for discrimination between elements that are residues of the pigment production processes and the elements that were introduced after the synthesis process, mainly as a result of contamination.

The mapping of impurities and inclusions within pigment particles, with the support of SEM measurements, provides information which, in some cases, allows for discrimination between different manufacturing routes. Data obtained in this work will inform the definition of elemental markers for future in situ analysis of paint samples, with implications for the analysis of $\mathrm{Zn}$-based whites from early $20^{\text {th }}$ century paintings. Future research should focus on the study of modifications in the optical properties of the pigments due to transition metal impurities and the characterization of Co-based salts in lithopone pigments.

\section{Acknowledgements}

Access to the SOLEIL Facility was funded through the CALIPSO European program. $^{30}$ Funding for research was provided through the FUTURAHMA project (http://www.futurahma.it), From Futurism to Classicism (1910-1922). Research, Art History and Materials Analysis. The research project FUTURAHMA (2013-2016) is financed by the Italian Ministry of Education, University and Research (MIUR) within the Future in Research 2012 program. We gratefully acknowledge the Fondazione Livorno for the sample from the Grubicy Benvenuti Collection, Prof. Aviva Burnstock from the Courtauld Institute of Art, and COLART for samples from the Lefranc Collection. Prof. Lucia
Toniolo of the Politecnico of Milan is thanked for providing other samples.

\section{Notes and references}

1 G. Osmond, J. Boon, L. Puskar and J. Drennan, Appl. Spectrosc., 2012, 66, 1136-1144.

2 D. Comelli, A. Nevin, A. Brambilla, I. Osticioli, G. Valentini, L. Toniolo, M. Fratelli and R. Cubeddu, Appl. Phys. A, 2011, 106(1), 25-34.

3 C. Clementi, F. Rosi, A. Romani, R. Vivani, B. G. Brunetti and C. Miliani, Appl. Spectrosc., 2012, 66(10), 1233-1241.

4 L. Bertrand, L. Robinet, M. Thoury, K. Janssens, S. X. Cohen and S. Schöder, Appl. Phys. A, 2011, 106(2), 377-396.

5 M. Cotte, J. Susini, J. Dik and K. Janssens, Acc. Chem. Res., 2010, 43(6), 705-714.

6 L. Monico, G. Van der Snickt, K. Janssens, W. De Nolf, C. Miliani, J. Verbeeck, H. Tian, H. Tan, J. Dik, M. Radepont and M. Cotte, Anal. Chem., 2011, 83(4), 12141223.

7 F. Casadio and V. Rose, Appl. Phys. A, 2013, 111(1), 1-8.

8 M. Thoury, J.-P. Echard, M. Réfrégiers, B. Berrie, A. Nevin, F. Jamme and L. Bertrand, Anal. Chem., 2011, 83(5), 17371745.

9 L. Bertrand, M. Réfrégiers, B. Berrie, J.-P. Echard and M. Thoury, Analyst, 2013, 138(16), 4463-4469.

10 A. Moezzi, A. M. McDonagh and M. B. Cortie, Chem. Eng. J., 2012, 185-186, 1-22.

11 G. Buxbaum and G. Pfaff, Industrial Inorganic Pigments, Wiley-vch, Germany, 2005.

12 C. D. Holley, The lead and zinc pigments, J. Wiley and Sons, California, USA, 1909.

13 N. Eastaugh, V. Walsh, T. Chaplin and R. Siddall, Pigment compendium: a dictionary and optical microscopy of historical pigments, Butterworth-Heinemann, Oxford, UK, 2004.

14 R. Capua, J. Am. Inst. Conserv., 2014, 53(2), 75-88.

15 G. Jantsch and P. Wolski, US pat. 1693902, United States Patent Office, 1928.

16 M. Bacci, M. Picollo, G. Trumpy, M. Tsukada and D. Kunzelman, J. Am. Inst. Conserv., 2007, 46(1), 27-37.

17 http://www.winsornewton.com/na/discover/articles-andinspiration/spotlight-on-chinese-white, accessed 02/10/ 2014.

18 A.-M. Flank, G. Cauchon, P. Lagarde, S. Bac, M. Janousch, R. Wetter, J.-M. Dubuisson, M. Idir, F. Langlois, T. Moreno and D. Vantelon, Nucl. Instrum. Methods Phys. Res., Sect. B, 2006, 246(1), 269-274.

19 V. a. Solé, E. Papillon, M. Cotte, P. Walter and J. Susini, Spectrochim. Acta, Part B, 2007, 62(1), 63-68.

20 B. Ravel and M. Newville, J. Synchrotron Radiat., 2005, 12, 537-541.

21 R. J. H. Clark, Spectrochim. Acta, Part A, 2001, 57(7), 14911521.

22 P. Vandenabeele, B. Wehling, L. Moens, H. Edwards, M. De Reu and G. Van Hooydonk, Anal. Chim. Acta, 2000, 407, 261274. 
23 D. M. McKnight, B. A. Kimball and R. L. Runkel, Hydrol. Processes, 2001, 15(10), 1979-1992.

24 A. Pantelouris, H. Modrow, M. Pantelouris, J. Hormes and D. Reinen, Chem. Phys., 2004, 300, 13-22.

25 H. Dourif, US pat. 1587704, United States Patent Office, 1926.

26 N. Heaton, Outlines of Paint Technology, Charles Griffin \& Co., London, 3rd edn, 1947.
27 P. Fleury, The preparation and uses of white zinc paints, Scott, Greenwood \& Son, London, 1912.

28 T. E. Westre, P. Kennepohl, J. G. Dewitt, B. Hedman, K. O. Hodgson and E. I. Solomon, J. Am. Chem. Soc., 1997, 119, 6297-6314.

29 D. L. A. de Faria, S. Venâncio Silva and M. T. de Oliveira, J. Raman Spectrosc., 1997, 28, 873-878.

30 http://www.calipso.wayforlight.eu/, accessed 02/10/2014. 